\title{
Hyperons - a strange key to the strong interaction
}

\author{
Karin Schönning $^{1 \star}$ on behalf of the BESIII and PANDA collaborations
}

1 Department of Physics and Astronomy, Uppsala University

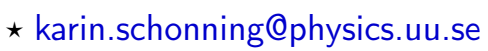

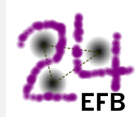

Proceedings for the 24th edition of European Few Body Conference,

Surrey, UK, 2-6 September 2019

doi:10.21468/SciPostPhysProc.3

\begin{abstract}
Hyperons provide new insights into two of the most challenging problems in contemporary physics: a coherent and quantitative description of the strong interaction, and the matter-antimatter asymmetry of the Universe. In these proceedings, I will demonstrate the merits of hyperons as a diagnostic tool using two recent measurements by the BESIII experiment as an illustrative example. Furthermore, I will highlight the prospect of future hyperon studies with the next-generation experiment PANDA at FAIR.
\end{abstract}

(c) (1) Copyright K. Schönning et al.

This work is licensed under the Creative Commons

Attribution 4.0 International License.

Published by the SciPost Foundation.
Received 28-11-2019

Accepted 27-01-2020

Published 25-02-2020

doi:10.21468/SciPostPhysProc.3.027

\section{Contents}

1 Introduction $\quad 2$

2 Hyperons 3

3 Hyperon Structure $\quad 4$

3.1 Previous measurements 5

3.2 New hyperon structure measurement with BESIII 6

3.2.1 The BESIII experiment 6

$\begin{array}{ll}3.2 .2 & \text { Analysis and formalism }\end{array}$

$\begin{array}{lll}3.2 .3 & \text { Results } & 6\end{array}$

$\begin{array}{lll}3.3 & \text { Future prospects } & 7\end{array}$

4 Hyperon Decays $\quad 7$

$\begin{array}{lll}4.1 & \text { CP test with BESIII } & 8\end{array}$

4.1.1 Formalism $\quad 8$

4.1.2 Recent measurement of $\Lambda$ decays $\quad 8$

$\begin{array}{lll}\text { 4.1.3 Future measurements with BESIII } & 9\end{array}$

5 Future hyperon physics with PANDA 9

5.1 The PANDA experiment 9 


\section{Introduction}

If I were to summarise the diversity of topics and discussions at the Few Body Conference with one over-arching theme, it would be nucleons. A large part of the community take an interest in how nucleons form bound systems and how they interact with other nucleons, either when being brought to collide in experiments, or in macroscopic objects such as stars. The hadron oriented community explore its building blocks, the quarks, and their interactions. Constituting the major part of the visible mass of Universe, nucleons earned their place in the spotlight a century after its discovery ${ }^{1}$. In fact, many of the most challenging questions in contemporary physics manifest themselves in our difficulties in understanding the nucleon features from first principles.

- Abundance: There is much more matter (nucleons) than anti-matter (anti-nucleons) in the Universe. If this was not fine-tuned at the Big Bang, then this asymmetry must be of dynamical origin [1].

- Spin: The sum of the measured valence quarks' contribution to the nucleon spin is only about half of the total spin [2].

- Mass: The nucleon mass is dynamically generated by the strong interaction and described in terms of effective degrees of freedom [3].

- Structure: The neutron has an intriguing charge distribution: negative in the centre and at the rim, positive in between [4]. For a ground-state wave function, this kind of wiggles is surprising.

- Size: The electric radius of the proton has been determined from electron-proton scattering, from the spectrum of electronic hydrogen and from the spectrum of muonic hydrogen. Until recently, most measurements using electrons resulted in a larger proton radius than the results obtained with muons $[5,6]$.

The abundance relates to the origin of the Universe and searches for physics beyond the Standard Model at the Precision Frontier. The spin, mass and structure are consequences of the non-perturbative nature of the strong interaction. In this sense, the study of these features approaches the Standard Model at the Low Energy Frontier. The proton radius puzzle has put lepton universality into question. However, in a recent measurement of electronic hydrogen, a smaller radius was obtained that was in agreement with measurements with muons [7]. The internal disagreements of the electron data remain to be understood. This is also discussed in other contributions to this conference, for example Ref. [8].

An often successful approach in physics to obtain clues about an enigmatic system is to make a change to the system and see how it reacts. For instance, one can [9]:

\footnotetext{
${ }^{1}$ Here, I refer to the discovery of the proton in 1919 by Rutherford - the neutron was discovered in 1932 by Chadwick.
} 


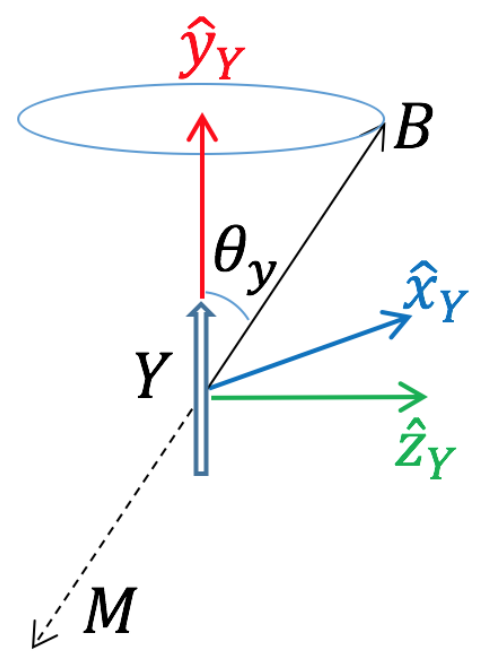

Figure 1: The $Y \rightarrow B M$ decay, with the spin direction of $Y$ along the $y$-axis.

\section{Scatter on it}

2. Excite it

3. Replace one of the building blocks

Starting with nucleons, we obtain hyperons through the third item: replacing a light quark by a strange, charm or bottom quark. In these proceedings, I will outline how different hyperon physics topics emerge from this approach and how they relate to the underlying, fundamental questions. Furthermore, I will discuss how these topics can be studied at existing facilities, e.g. BESIII, and future facilities, e.g. PANDA.

\section{Hyperons}

Hyperons are suitable for studying the strong interaction at the scale where quarks form hadrons. The minimum scale probed in a given reaction depends on the mass of the interacting entities. For strange hyperons, the scale is governed by the mass of the strange quark, $m_{s} \approx 95 \mathrm{MeV} / \mathrm{c}^{2}$. This is close to the cut-off $\Lambda_{Q C D} \approx 200 \mathrm{MeV} / \mathrm{c}^{2}$, defining the scale where quarks are confined into hadrons. As a consequence, the relevant degrees of freedom of such processes are unclear: quarks and gluons, or hadrons? Strange hyperons therefore probe the confinement domain of the strong interaction. This is in stark contrast to the more than ten times heavier charm quark $\left(m_{c} \approx 1275 \mathrm{MeV} / c^{2}\right)$, that probes the scale where perturbative QCD breaks down. Processes involving charmed hyperons can be used to bridge the transition region between perturbative and non-perturbative QCD.

Hyperons have an advantage compared to nucleons: their spin is traceable through their self-analysing decays. Weak decay amplitudes can have a parity conserving and a parity violating part. As a consequence, the daughter particles from a hyperon decay are emitted according to the direction of the spin of the mother hyperon. Figure 1 illustrates the two-body decay $Y \rightarrow B M$, where a spin $1 / 2$ hyperon $Y$ decays into a spin $1 / 2$ baryon $B$ and a pseudo-scalar meson $M$. The angular distribution $W\left(\cos \theta_{B}\right)$ of $B$ in the rest system of $Y$ can be expressed in terms of the polarisation $P_{y}\left(\cos \theta_{Y}\right)$ with respect to some reference axis $\vec{y}$ as a function of the $Y$ scattering angle $\theta_{Y}[10,11]$

$$
W\left(\cos \theta_{B}\right)=\frac{1}{4 \pi}\left(1+\alpha P_{y}\left(\cos \theta_{Y}\right) \cos \theta_{B}\right)
$$


$P_{y}$ carries information about the production process and depends on the energy. The decay asymmetry $\alpha$ is independent of the production mechanism and instead related to the interference between the parity violating and the parity conserving decay amplitudes, $T_{s}$ and $T_{p}[12]$. Equation 1 shows how parameters with physical meaning can be extracted from measurable quantities and demonstrates the unique potential of the hyperon as a diagnostic tool.

In Figure 2, we illustrate how hyperons connect two fundamental questions, resulting in four emerging research topics. In these proceedings, we will focus on hyperon structure and hyperon decays.

\section{Fundamental Question}

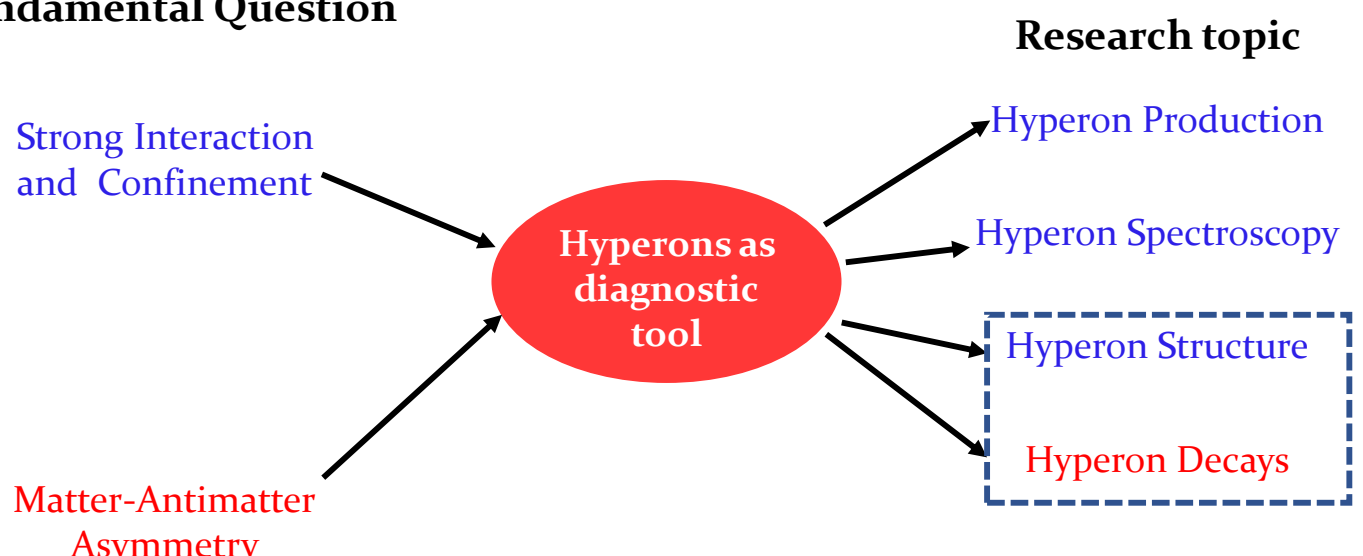

Figure 2: Conceptual map of hyperon physics topics, showing how the two overarching fundamental questions (left) can be approached by a common diagnostic tool, i.e. hyperons (middle). From this, the different subtopics emerge (right). The colour-coding indicates which questions are related to which research and the dashed rectangle marks the focus of these proceedings.

\section{Hyperon Structure}

The electromagnetic structure of hadrons is probed in interactions between hadrons and virtual photons. ElectroMagnetic Form Factors (EMFFs) describe the inner, dynamical structure and quantify the deviation from a point-like state. EMFFs are functions of the momentum transfer squared, $q^{2}$, carried by the virtual photon. As fundamental observables of the strong interaction, theoretical predictions of EMFFs can be made using e.g. Chiral Perturbation Theory $[13,14]$ or Lattice QCD [15-17].

EMFF's can be space-like ( $q^{2}<0$, to the left in Fig. 3), studied in elastic electron-hadron scattering, or time-like ( $q^{2}>0$, to the right of the $y$-axis in Fig. 3). The electric and the magnetic form factors in the space-like region have an intuitive interpretation since, in the so-called Breit frame, they are the Fourier transforms of the charge and magnetisation densities $[4,18]$. Unfortunately, the space-like region is difficult to access experimentally for the unstable hyperons, since they are unfeasible as beams or targets in electron-hyperon scattering experiments. Instead, time-like form factors constitute the most viable structure observables for hyperons [19].

In the time-like region, the EMFFs can be complex with a relative phase. This phase reflects the fluctuation of the virtual photon into intermediate hadron-antihadron states whose amplitudes interfere. A non-zero phase polarises effect on the final state hyperons, even if the 
initial state is unpolarised [20].

From the experimentally accessible time-like form factors, the more intuitive space-like form factors can be calculated using dispersion relations. For example, the asymptotic behavior of the time-like EMFFs as $q^{2} \rightarrow \infty$ can be obtained from the corresponding space-like region as a consequence of the Phragmén-Lindelöf theorem [21]. Above a certain scale where $q^{2}$ is large, the space-like and the time-like form factors should approach the same value. For nucleons, being accessible in the space-like as well as in the time-like region, the onset of this scale is straight-forward to test. For hyperons, we must proceed differently. Since we know that in the space-like region, EMFFs are real, we infer that the same must be true in the time-like region when $q^{2} \rightarrow \infty$. This means that the phase must be an integer multiple of $\pi$, depending on the $q^{2}$-power-law behaviour $[22,23]$ and the possible presence of spacelike zeros [19]. However, for intermediate $q^{2}$, the phase can be non-zero, which results in polarised hyperons and antihyperons in the final state. Thanks to the self-analysing decays of hyperons, the polarisation is experimentally accessible. Hence, polarised hyperons offer an alternative way to study the asymptotic behaviour of hyperon form factors: by measuring the phase at different $q^{2}$, the onset of the scale at which the hyperon time-like EMFFs approach the space-like can be established.

The time-like region can be further divided into two distinct parts with respect to the momentum transfer squared, $q^{2}$ :

- The high- $q^{2}$ part, explored when an $e^{+} e^{-}$pair annihilates to form a hyperon-antihyperon pair $\left(e^{+} e^{-} \rightarrow Y_{1} \bar{Y}_{2}\right.$, see Figure 2 a). This region covers momentum transfers larger than the sum of the masses of the produced hyperons $\left(q^{2}>\left(m_{Y_{1}}+m_{Y_{2}}\right)^{2}\right)$.

- The low- $q^{2}$ part, probed in hyperon Dalitz decays $\left(Y_{1} \rightarrow Y_{2} e^{+} e^{-}\right.$, see Figure $2 \mathrm{~b}$ ) and covering momentum transfers below the difference in mass between the hyperons $\left(q^{2}<\left(m_{Y_{1}}-m_{Y_{2}}\right)^{2}\right)$.

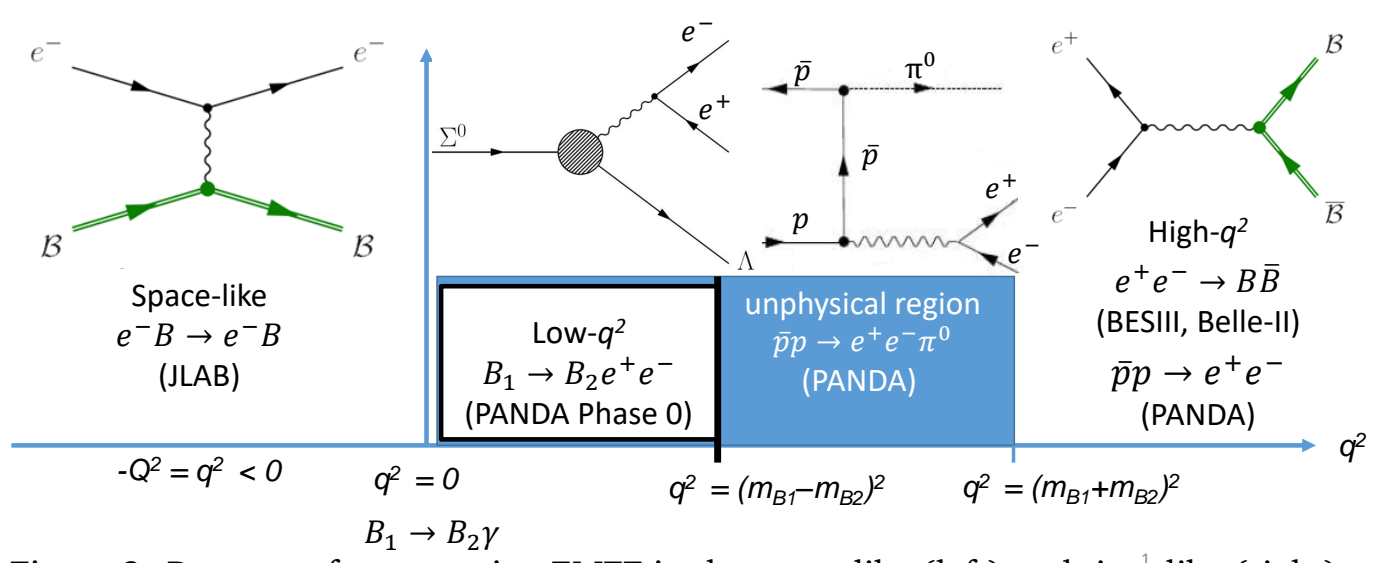

Figure 3: Processes for extracting EMFF in the space-like (left) and time-like (right) region. The low- $q^{2}\left(4 m_{e}^{2}<q^{2}<\left(M_{B 1}-M_{B 2}\right)^{2}\right)$ part of the time-like region is studied by Dalitz decays, the unphysical region $\left(4 m_{e}^{2}<q^{2}<\left(M_{B 1}+M_{B 2}\right)^{2}\right)$ by $\bar{p} p \rightarrow \ell^{+} \ell^{-} \pi^{0}$ and the high- $q^{2}$ region $\left(q^{2}>\left(M_{B 1}+M_{B 2}\right)^{2}\right)$ by $B \bar{B} \leftrightarrow e^{+} e^{-}$. Note that the unphysical region is only accessible for protons.

The different $q^{2}$ regions with their corresponding form factors and the processes where they are accessed, are shown in Fig. 3.

\subsection{Previous measurements}

So far, only a few measurements of hyperon form factors exist and they are all in the high$q^{2}$ region. Cross section measurements of the $e^{+} e^{-} \rightarrow Y \bar{Y}$ process were studied for different 
hyperons by BaBar [24], CLEO-c [25,26] and BESIII [27] but the data samples were too small to separate the electric and the magnetic form factor with any conclusive precision. Some information about the structure of $\Lambda_{c}^{+}$has been obtained by measurement by Belle [28] and BESIII [29].

\subsection{New hyperon structure measurement with BESIII}

\subsubsection{The BESIII experiment}

The Beijing Spectrometer (BESIII) experiment has recently carried out the first complete measurement of the time-like structure of any baryon. The BESIII detector is an integrated part of the Beijing Electron Positron Collider (BEPC-II) [30] and has a geometrical acceptance of 93\% of the solid angle. BESIII comprises a small-cell, helium-based main drift chamber (MDC), a time-of-flight system (TOF) based on plastic scintillators, an electromagnetic calorimeter (EMC) made of CsI(Tl) crystals, a muon counter (MUC) made of resistive plate chambers, and a superconducting solenoid magnet with a central field of 1.0 Tesla. A detailed description of the detector and its performance can be found in Ref. [31].

\subsubsection{Analysis and formalism}

In this work, the reaction $e^{+} e^{-} \rightarrow \Lambda \bar{\Lambda},\left(\Lambda \rightarrow p \pi^{-}, \bar{\Lambda} \rightarrow p \bar{\pi}^{+}\right)$has been studied. A dedicated data sample corresponding to an integrated luminosity of $66.9 \mathrm{pb}^{-1}$ was collected for this purpose at a CMS energy of $q=2.396 \mathrm{GeV}$. The analysis, including event selection, efficiency correction and background treatment, follows common standards and is outlined in Ref. [32].

A complete decomposition of the complex form factors $G_{E}$ and $G_{M}$ requires a multi-dimensional analysis of the reaction and the subsequent decays. In Refs. [33,34], the joint angular distribution of $e^{+} e^{-} \rightarrow \Lambda \bar{\Lambda},\left(\Lambda \rightarrow p \pi^{-}, \bar{\Lambda} \rightarrow \bar{p} \pi^{+}\right)$was derived in terms of the phase $\Delta \Phi$ and the angular distribution parameter $\eta=\left(\tau-R^{2}\right) /\left(\tau+R^{2}\right)$, where $\tau=q^{2} /\left(4 m_{Y}^{2}\right)$ and $R=\left|G_{E} / G_{M}\right|$. The differential cross section of the full process depends on five angles. In particular, the transverse polarisation of $\Lambda, P_{y}$, can be expressed in terms of the phase $\Delta \Phi$ and the $\Lambda$ scattering angle:

$$
P_{y}=\frac{\sqrt{1-\eta^{2}} \sin \theta \cos \theta}{1+\eta \cos ^{2} \theta} \sin (\Delta \Phi) \text {. }
$$

\subsubsection{Results}

The phase $\Delta \Phi$ and the angular distribution parameter $\eta$ were estimated by fitting the fivedimensional angular distribution from Ref. [34] to the data using a Maximum Log Likelihood fit. From this, the form factor ratio could be estimated to be $R=0.96 \pm 0.14 \pm 0.02$, and the phase to be $\Delta \Phi=37^{\circ} \pm 12^{\circ} \pm 6^{\circ}$. The first uncertainty is statistical and the second is systematic. The latter is estimated from a thorough investigation of possible sources, where the uncertainties from luminosity, tracking and background are found to be negligible. Nonnegligible contributions arise from the fit range and selection criteria. We find that the total systematic uncertainty is about seven times smaller than the statistical for $R$ and about two times smaller for $\Delta \Phi$. The correlation coefficient between $\eta$ and $\Delta \Phi$ is 0.17 .

The non-zero value of the relative phase implies that the imaginary part of the electric and the magnetic form factors are different. Equivalently, this means that not only the $s$-wave but also the $d$-wave amplitude contribute to the production and their interference results in a polarised final state.

In addition, the cross section was obtained to be $\sigma=118.7 \pm 5.3$ (stat.) \pm 5.1 (sys.) pb. From this, the effective form factor could be extracted to be $|G|=0.123 \pm 0.003$ (stat.) \pm 0.003 (sys.). This is about one half of that of the proton at the corresponding excess energy [35]. 
From our measurements, we can also learn about the $\Lambda \bar{\Lambda}$ interaction close to threshold. In a recent theory paper [36], predictions were made using Final State Interaction (FSI) potentials [37] from fits to PS185 data from the $\bar{p} p \rightarrow \Lambda \bar{\Lambda}$ reaction [38]. In their work, the sensitivity of $R$ and $\Delta \Phi$ to the FSI potential was found to be much more pronounced than that of the total cross section. Our measurement slightly favors the Model I or Model II potential of Ref. [37].

\subsection{Future prospects}

This first complete hyperon EMFF measurement is a milestone in the study of hyperon structure, where the long-term goal is to describe charge and magnetization densities for hyperons in the same way as for nucleons [4]. In order to achieve this, similar measurements must be carried out at several energies. For this purpose, the methods developed for this study can be applied at other energies, provided the data sample at each energy is large enough. Further measurements can be performed by BESIII (strange and single charm hyperons) and Belle II (primarily charmed hyperons).

In the near future, it will also be possible to carry out pioneering measurements in the low$q^{2}$ region from hyperon Dalitz decays with the HADES-PANDA setup. This effort, that combines the excellent di-electron detection capability of HADES with the tracking of hyperon decay products with forward tracker planes from PANDA, is a part of the FAIR Phase 0 campaign [39].

\section{Hyperon Decays}

According to today's paradigm, equal amounts of matter and antimatter were created in the Big Bang. However, from observations we know that the visible Universe consists of matter, not antimatter. Where did the antimatter go? The dynamical enrichment of matter with respect to antimatter is called Baryogenesis [1]. It is only possible if the following criteria are fulfilled:

- Processes exist which violate baryon number conservation.

- Processes exist which violate charge conjugation (C) and charge conjugation and parity (CP) symmetry.

- The processes above have occurred outside thermal equilibrium.

$\mathrm{CP}$ violation is possible in weak interactions by the Cabibbo-Kobayashi-Maskawa mechanism $[40,41]$, now an inherent part of the SM. Numerous CP violating effects in $K_{0}$ and $B$ meson decays have been found by BaBar, Belle and at Fermilab and CERN [42]. Very recently, the LHCb collaboration reported the first evidence of CP violation in decays of the charmed $D$ meson [43]. However, since our visible Universe consists largely of spin carrying baryons, it is of highest relevance to look for $\mathrm{CP}$ violation also in baryon decays. The only indication of $\mathrm{CP}$ violating baryon decay observed so far was seen in the four-body decay of the bottom $\Lambda_{b}$ hyperon in LHCb [44]. The deviation is consistent with the SM. In total, no CP violating effects beyond the SM have ever been seen, neither in the meson nor in the baryon sector. Furthermore, all deviations are too small to explain the observed matter-antimatter asymmetry of the Universe. Indeed, Baryogenesis requires physics beyond the SM [45].

In the strange baryon sector, predictions from SuperSymmetry (SuSy) [46] indicate CP violating effects that are up to two orders of magnitude larger than in the SM [47]. Precise measurements of hyperon decays can therefore put SM and beyond SM predictions to the test. Strange hyperons also have the advantage that they predominantly decay into two-body states for which CP-odd observables are straight-forward to define. CP conservation means that particles and antiparticles have the same decay pattern. Hence for a weak, two-body 
hyperon decay, the asymmetry parameter $\alpha$ in Eq. 1 should be the same as the $-\bar{\alpha}$ of the corresponding antihyperon. Then one can define

$$
A_{C P}=\frac{\alpha+\bar{\alpha}}{\alpha-\bar{\alpha}}
$$

for which a non-zero value indicates $\mathrm{CP}$ violation [10].

In the case of multi-strange hyperons, e.g. the $\Xi^{-}$, the sequential weak decays provide access to the additional decay asymmetry parameters $\beta$ and $\phi$. From $\beta$ and $\bar{\beta}$, the asymmetries $B_{C P}, B_{C P}^{\prime}$ and $\Delta \phi$ can be defined in a similar manner as for $A_{C P}$ in Eq. 3 . These are more sensitive to $C P$ violation by a factor of 100 and 10, respectively [48]. The most precise experimental CP test for strange baryons so far is provided by the HyperCP experiment at Fermilab and concerns the sequential decay of the double-strange $\Xi^{-}$hyperon [49].

\subsection{CP test with BESIII}

In BESIII, hyperon decays can be studied in processes like $e^{+} e^{-} \rightarrow J / \Psi \rightarrow Y \bar{Y}, Y \rightarrow B M$, $\bar{Y} \rightarrow \bar{B} M$. Thanks to the relatively large branching fractions of $J / \Psi$ decays into single- and double hyperon-antihyperon pairs $\left(B R \approx 10^{-3}\right)$, we can benefit from the large $J / \Psi$ samples collected by BESIII $\left(1.2 \cdot 10^{9}\right.$ in 2012 and $10^{10}$ in 2018).

\subsubsection{Formalism}

Most experiments with hadronic probes apply a formalism that only considers the decays, not pinning down the production process. In such an approach, one integrates over all possible spin directions. This simplifies the formulas but leads to loss of information and hence worse precision. In particular, the detector efficiency, typically evaluated from Monte Carlo simulations, is model dependent since it depends on the angular distributions of the generated particles. This introduces a systematic bias for any non-ideal detector that does not cover $100 \%$ of the full $4 \pi$ solid angle. Hyperon-antihyperon production in $e^{+} e^{-}$colliders have the advantage that the production is dominated by photon or vector meson exchange. Therefore, in the production of spin $\frac{1}{2}$ hyperons, only $s$ and $d$ partial waves are expected to contribute. This means that the full production and decay process of weakly decaying, single-strange hyperons can be parameterised in terms of two production parameters, e.g. the angular distribution parameter $\eta$ and the phase $\Delta \Phi$, and two decay parameters $\alpha_{Y}$ and $\bar{\alpha}_{\bar{Y}}$. The multi-dimensional formalism outlined in Refs. $[33,34]$ provides a model-independent approach since the only assumption is that the process is dominated by vector exchange. Since the full production and decay process is parameterised, the use of measured information is maximised. As a consequence, the precision for a given sample size is increased.

\subsubsection{Recent measurement of $\Lambda$ decays}

The method was implemented in the analysis of $J / \Psi \rightarrow \Lambda \bar{\Lambda}\left(\Lambda \rightarrow p \pi^{-}, \bar{\Lambda} \rightarrow \bar{p} \pi^{+} / \bar{\Lambda} \rightarrow \bar{n} \pi^{0}\right)$, described in detail in Ref. [50]. The purely charged mode ( $p \pi^{-} \bar{p} \pi^{+}$final state) comprised 420000 fully reconstructed events and the charged-neutral ( $p \pi^{-} \bar{n} \pi^{0}$ final state) 47000 events. The phase $\Delta \Phi$ was extracted from the two modes simultaneously and was found to be $\Delta \Phi=42.4^{\circ} \pm 0.6^{\circ} \pm 0.05^{\circ}$. The decay asymmetry $\alpha_{-}$for the $\Lambda \rightarrow p \pi^{-}$decay was obtained to be $0.750 \pm 0.009 \pm 0.004$. This is $17 \pm 3 \%$ larger than the previously established value of $0.642 \pm 0.013$ obtained from a series of experiments in the 1960s and 1970s [51-55]. In these experiments, a different method was used: instead of extracting the decay asymmetry from the $\Lambda$ decay distribution, it was obtained from the the measured polarisation of the daughter proton. It is difficult to explain the discrepancy in the results since the older papers discuss 
systematics only briefly. It is also noteworthy that the data samples in previous measurements were about 40 times smaller compared to the recent BESIII sample. The new value, obtained by BESIII, has been accepted by the Particle Data Group in the 2019 update of Ref. [11]. The revision has a large impact on the field of hyperon polarisation physics as a whole. In most experiments, the measured quantity is the product $\alpha_{-} P_{y}$ rather than the polarisation $P_{y}$. The latter is instead calculated from the product using the PDG value of $\alpha_{-}$as input. The updated $\alpha_{-}$means that all measured values of the polarisation so far must be downscaled by $17 \%$.

In addition to the $\alpha_{-}$measurement, $\alpha_{+}$was measured to be $-0.758 \pm 0.010 \pm 0.007$ and $\alpha_{0}$ of the $\bar{\Lambda} \rightarrow \bar{n} \pi^{0}$ decay to be $-0.692 \pm 0.016 \pm 0.006$. From $\alpha_{-}$and $\alpha_{+}$, the CP odd asymmetry $A_{C P}$, defined in Eq. 3, was calculated to be $-0.006 \pm 0.012 \pm 0.007$. This is the most precise CP test of the $\Lambda$ hyperon decay so far.

\subsubsection{Future measurements with BESIII}

The world-record sample of $10^{10} \mathrm{~J} / \Psi$ events collected in 2018 by BESIII opens up unprecedented possibilities. In particular, hitherto unexplored aspects of sequentially decaying $\Sigma^{0}, \Xi^{-}$ and $\Xi^{0}$ can be studied. The formalism for decays of charged and neutral $\Xi$ have been outlined in Ref. [56] and that of $\Sigma^{0}$ in Ref. [57]. The latter is an electromagnetic decay and is therefore very unlikely to violate CP symmetry [58]. However, this means that even small signals would indicate new physics.

\section{Future hyperon physics with PANDA}

The recent, ongoing and future CP tests with BESIII are important milestones in the search for $\mathrm{CP}$ violation and thereby gain insights about the matter-antimatter asymmetry of the Universe. However, the data samples that can be achieved will only provide a precision of at most $10^{-3}$. To test predictions of the SM and beyond, the precision needs to be improved by at least two orders of magnitude. Among the currently operating or planned facilities world-wide, the anti-Proton ANnihiltions in DArmstadt (PANDA), at the future Facility for Antiproton and Ion Research, is the most suitable for this task.

\subsection{The PANDA experiment}

In PANDA, hyperon-antihyperon pairs will be produced in antiproton-proton annihilations. The antiprotons will be delivered from the High Energy Storage Ring (HESR) within a momentum range from $1.5 \mathrm{GeV} / c$ up to $15 \mathrm{GeV} / c$. During the start-up, referred to as Phase One, the HESR will be able to accumulate up to $10^{10}$ antiprotons within a time span of $1000 \mathrm{~s}$. In a final stage, Phase Three, the dedicated Recuperated Experimental Storage Ring (RESR) will allow up to $10^{11}$ antiprotons to be injected and stored in the HESR. In the HESR, the beam will be stochastically cooled which will enable a beam momentum spread of better than $5 \cdot 10^{-5}$. The antiproton beam will impinge on a hydrogen cluster jet or pellet target. During Phase One, the HESR will provide a luminosity of $\approx 10^{31} \mathrm{~cm}^{2} \mathrm{~s}^{-1}$ whilst the design luminosity of $\approx 2 * 10^{32} \mathrm{~cm}^{2} \mathrm{~s}^{-1}$ will be achieved during Phase Three.

The near $4 \pi$ solid angle coverage of the PANDA detector will provide exclusive reconstruction of the final state particles, minimising systematic effects from e.g. efficiency. PANDA will consist of a target spectrometer part (TS) and a forward spectrometer part (FS). The TS provides precise vertex tracking by the micro vertex detector (MVD), straw tube trackers (STT) and gas electron multiplier detectors (GEM). Time-of-flight detectors (TOF) and detection of internally reflected Cherenkov light (DIRC) offer particle identification and an electromagnetic calorimeter (EMC) will measure energies. The trajectories in the TS are bent by the field of a 
solenoid magnet providing a field of 2.0 T. The FS consists of straw tube stations for tracking, a dipole magnet, a ring imaging Cherenkov counter (RICH) detector and a TOF for particle identification and a Shashlyk electromagnetic calorimeter. The PANDA detector is described in further detail in Ref. [59].

\subsection{Prospects for hyperon physics with PANDA}

The fact that hyperons and antihyperons can be produced in two-body reactions is an advantage compared to meson or photon probes, where strangeness conservation requires that the hyperon is produced with the corresponding number of associated kaons. Such manybody final states are difficult to parameterise in terms of partial waves and hence a modelindependent approach, such as the one outlined in Section 3.2.2, is no longer possible. The two-body, particle-antiparticle symmetric final state in PANDA is more straight-forward to parameterise. Therefore it provides excellent conditions for the planned hyperon physics programme that includes hyperon production, hyperon structure, hyperon spectroscopy and, in Phase Three, CP tests in hyperon decays.

The main advantage compared to $e^{+} e^{-}$colliders are the large cross sections for hyperonantihyperon pair production. Existing data from single- and double-strange hyperons [60] indicate cross sections within and above the microbarn range, as shown in Figure 4.
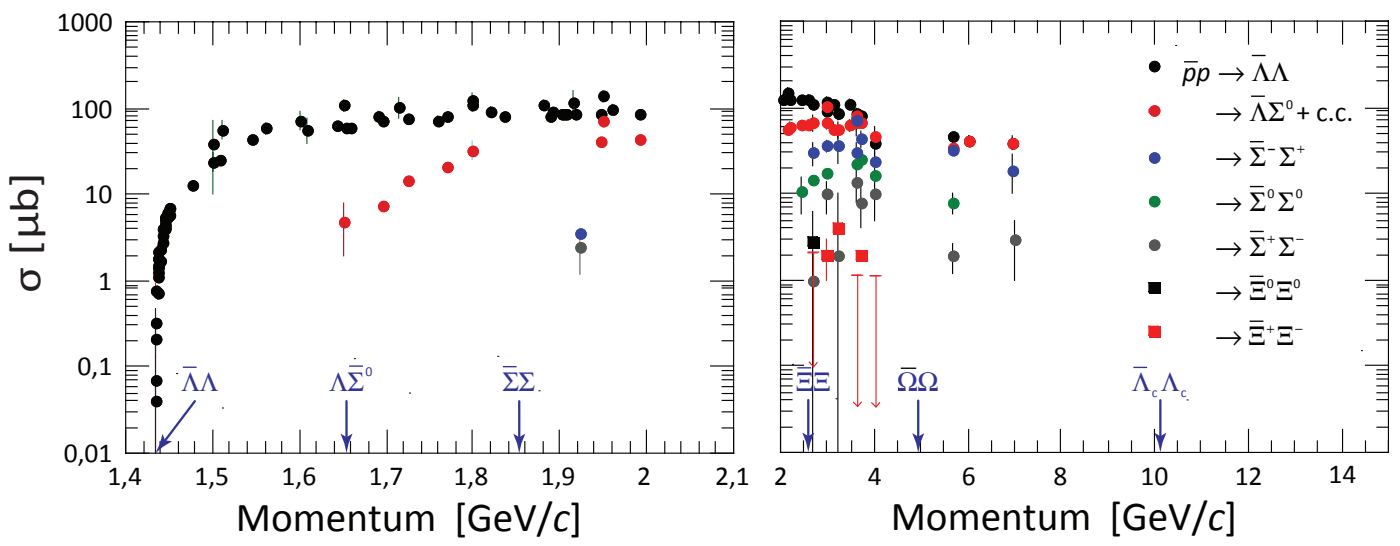

Figure 4: The cross sections of various hyperon-antihyperon pairs produced in antiproton-proton annihilations. The data are primarily from the PS185 experiment at LEAR and compiled in Ref. [60].

In order to investigate the prospects of hyperon physics with PANDA in more detail, simulation studies have been performed in a recent campaign. The following processes were studied:

- $\bar{p} p \rightarrow \bar{\Lambda} \Lambda, \bar{\Lambda} \rightarrow \bar{p} \pi^{+}, \Lambda \rightarrow p \pi^{-}$at $p_{\bar{p}}=1.64 \mathrm{GeV} / \mathrm{c}[61]$,

- $\bar{p} p \rightarrow \bar{\Sigma}^{0} \Lambda, \bar{\Sigma}^{0} \rightarrow \bar{\Lambda} \gamma, \bar{\Lambda} \rightarrow \bar{p} \pi^{+}, \Lambda \rightarrow p \pi^{-}$at $p_{\bar{p}}=1.77$ and $6.0 \mathrm{GeV} / \mathrm{c}$ [62],

- $\bar{p} p \rightarrow \bar{\Xi}^{+} \Xi^{-}, \bar{\Xi}^{+} \rightarrow \bar{\Lambda} \pi^{+}, \Xi^{-} \rightarrow \Lambda \pi^{-}$at $p_{\bar{p}}=4.6$ and $7.0 \mathrm{GeV} / \mathrm{c}[61]$.

The simulations were performed using the PandaROOT framework [63], with ideal pattern recognition and particle identification. Additional selection criteria were applied that mimic the effects from a full, realistic implementation. The resulting signal efficiencies are given in Table 1 and show that the exclusive hyperon reconstruction rates will be high. Combined with the low background level, this demonstrates the potential of PANDA as a hyperon factory. 
Table 1: Results from simulation studies of the various production reactions of ground state hyperons $[61,62]$. The efficiencies are for exclusive reconstruction, i.e. considering all final state particles. The $S / B$ denotes signal-to-background ratio and the asterisk * the upper limit of a $90 \%$ confidence interval.

\begin{tabular}{llllllll}
\hline$p_{\bar{p}}(\mathrm{GeV} / c)$ & Reaction & $\sigma(\mu \mathrm{b})$ & Eff (\%) & Decay & $\mathrm{S} / \mathrm{B}$ & $\begin{array}{l}\text { Rate }\left(s^{-1}\right) \\
\text { at } 10^{31} \\
\mathrm{~cm}^{-2} \mathrm{~s}^{-1}\end{array}$ & $\begin{array}{l}\text { Rate }\left(s^{-1}\right) \\
\text { at } 2 \cdot 10^{32} \\
\mathrm{~cm}^{-2} \mathrm{~s}^{-1}\end{array}$ \\
\hline 1.64 & $\bar{p} p \rightarrow \bar{\Lambda} \Lambda$ & $64.0[38]$ & $15.7[61]$ & $\Lambda \rightarrow p \pi^{-}$ & $114[61]$ & 44 & 880 \\
\hline 1.77 & $\bar{p} p \rightarrow \bar{\Sigma}^{0} \Lambda$ & $10.9[38]$ & $5.3[62]$ & $\Sigma^{0} \rightarrow \Lambda \gamma$ & $>11^{*}[62]$ & 2.4 & 48 \\
\hline 6.0 & $\bar{p} p \rightarrow \bar{\Sigma}^{0} \Lambda$ & $20.0[64]$ & $6.1[62]$ & $\Sigma^{0} \rightarrow \Lambda \gamma$ & $21[62]$ & 5.0 & 100 \\
\hline 4.6 & $\bar{p} p \rightarrow \bar{\Xi}^{+} \Xi^{-}$ & $1.0[65]$ & $8.2[61]$ & $\Xi^{-} \rightarrow \Lambda \pi^{-}$ & $274[61]$ & 0.3 & 6 \\
\hline 7.0 & $\bar{p} p \rightarrow \bar{\Xi}^{+} \Xi^{-}$ & $0.3[65]$ & $7.9[61]$ & $\Xi^{-} \rightarrow \Lambda \pi^{-}$ & $165[61]$ & 0.1 & 2 \\
\hline
\end{tabular}

\section{Conclusion}

Hyperons provide a powerful diagnostic tool that sheds light on two of the most challenging problems in contemporary physics: the strong interaction in the confinement domain and the matter-antimatter asymmetry of the Universe. In particular, its experimentally accessible spin properties provide new insights into hyperon structure as well as clean tests of CP violation in hyperon decays. New measurements from BESIII provide important milestones in understanding hyperon structure and decay. The future PANDA experiment at FAIR will be a veritable hyperon factory, where many aspects of hyperon physics can be studied with unprecedented precision. Already in 2020-2021, it will be possible to perform pioneering measurements of the low-energy structure of hyperons through the FAIR Phase 0 initiative HADES/PANDA.

\section{Acknowledgements}

This work was supported by the Knut and Alica Wallenberg Foundation (Contract No. 2016.0157), the Swedish Research Council (No. 2013-04278) and STINT (No. CH2018-7756).

\section{References}

[1] A. D. Sakharov, Violation of CP invariance, $C$ asymmetry, and baryon asymmetry of the universe, Sov. Phys. Usp. 34, 392 (1991), doi:10.1070/PU1991v034n05ABEH002497.

[2] C. A. Aidala, S. D. Bass, D. Hasch and G. K. Mallot, The spin structure of the nucleon, Rev. Mod. Phys. 85, 655 (2013), doi:10.1103/RevModPhys.85.655.

[3] M. R. Schindler, D. Djukanovic, J. Gegelia and S. Scherer, Infrared renormalization of two-loop integrals and the chiral expansion of the nucleon mass, Nucl. Phys. A 803, 68 (2008), doi:10.1016/j.nuclphysa.2008.01.023.

[4] G. A. Miller, Charge densities of the neutron and proton, Phys. Rev. Lett. 99, 112001 (2007), doi:10.1103/PhysRevLett.99.112001.

[5] R. Pohl et al., The size of the proton, Nature 466, 213 (2010), doi:10.1038/nature09250. 
[6] C. E. Carlson, The proton radius puzzle, Prog. Part. Nucl. Phys. 82, 59 (2015), doi:10.1016/j.ppnp.2015.01.002.

[7] N. Bezginov, T. Valdez, M. Horbatsch, A. Marsman, A. C. Vutha and E. A. Hessels, A measurement of the atomic hydrogen Lamb shift and the proton charge radius, Science 365, 1007 (2019), doi:10.1126/science.aau7807.

[8] S. Bacca, Muonic Lithium atoms: nuclear structure corrections to the Lamb shift, SciPost Phys. Proc. 3, 028 (2020), doi:10.21468/SciPostPhysProc.3.028.

[9] C. Granados, S. Leupold and E. Perotti, The electromagnetic Sigma-to-Lambda hyperon transition form factors at low energies, Eur. Phys. J. A 53, 117 (2017), doi:10.1140/epja/i2017-12324-4.

[10] I. I. Bigi, A. I. Sanda, CP violation Cambridge Monographs on Particle Physics, Nuclear Physics and Cosmology, 9, Cambridge University Press (2000).

[11] M. Tanabashi et al., Review of particle physics, Phys. Rev. D 98, 030001 (2018), doi:10.1103/PhysRevD.98.030001.

[12] T. D. Lee and C. N. Yang, General partial wave analysis of the decay of a hyperon of spin 1/2, Phys. Rev. 108, 1645 (1957), doi:10.1103/PhysRev.108.1645.

[13] S. Weinberg, Effective chiral lagrangians for nucleon-pion interactions and nuclear forces, Nucl. Phys. B 363, 3 (1991), doi:10.1016/0550-3213(91)90231-L.

[14] R. Machleidt and D. R. Entem, Chiral effective field theory and nuclear forces, Phys. Rep. 503, 1 (2011), doi:10.1016/j.physrep.2011.02.001.

[15] S. Boinepalli, D. B. Leinweber, A. G. Williams, J. M. Zanotti and J. B. Zhang, Precision electromagnetic structure of octet baryons in the chiral regime, Phys. Rev. D 74, 093005 (2006), doi:10.1103/PhysRevD.74.093005.

[16] H-W. Lin and K. Orginos, Strange baryon electromagnetic form factors and SU(3) flavor symmetry breaking, Phys. Rev. D 79, 074507 (2009), doi:10.1103/PhysRevD.79.074507;

[17] P. E. Shanahan et al., Magnetic form factors of the octet baryons from lattice QCD and chiral extrapolation, Phys. Rev. D 89, 074511 (2014), doi:10.1103/PhysRevD.89.074511.

[18] V. Punjabi, C. F. Perdrisat, M. K. Jones, E. J. Brash and C. E. Carlson, The structure of the nucleon: Elastic electromagnetic form factors, Eur. Phys. J. A 51, 79 (2015), doi:10.1140/epja/i2015-15079-x.

[19] S. Pacetti, R. Baldini Ferroli and E. Tomasi-Gustafsson, Proton electromagnetic form factors: Basic notions, present achievements and future perspectives, Phys. Rep. 550-551, 1 (2015), doi:10.1016/j.physrep.2014.09.005.

[20] A. Z. Dubničková, S. Dubnička and M. P. Rekalo, Investigation of the baryon electromagnetic structure by polarization effects in $e^{+} e^{-} \rightarrow B \bar{B}$ processes, Nuov. Cim. A 109, 241 (1996), doi:10.1007/BF02731012.

[21] E. C. Titschmarsh, The theory of functions, Oxford University Press (1939).

[22] V. A. Matveev, R. M. Muradyan and A. N. Tavkhelidze, Scaling in strong interaction, Theor. Mat. Fiz. 15, 332 (1973). 
[23] S. J. Brodsky and G. R. Farrar, Scaling laws at large transverse momentum, Phys. Rev. Lett. 31, 1153 (1973), doi:10.1103/PhysRevLett.31.1153.

[24] B. Aubert et al., Study of $e^{+} e^{-} \rightarrow \Lambda \bar{\Lambda}, \Lambda \bar{\Sigma}^{0}$, and $\Sigma^{0} \bar{\Sigma}^{0}$ using initial state radiation with $B A B A R$, Phys. Rev. D 76, 092006 (2007), doi:10.1103/PhysRevD.76.092006.

[25] S. Dobbs, A. Tomaradze, T. Xiao, K. K. Seth and G. Bonvicini, First measurements of timelike form factors of the hyperons $\Lambda, \Sigma^{0}, \Sigma^{+}, \Xi^{0}, \Xi^{-}$, and $\Omega^{-}$, and evidence of diquark correlations, Phys. Lett. B 739, 90 (2014), doi:10.1016/j.physletb.2014.10.025.

[26] S. Dobbs et al., Hyperon form factors and diquark correlations, Phys. Rev. D 96, 092004 (2017), doi:10.1103/PhysRevD.96.092004.

[27] M. Ablikim et al., Observation of a cross-section enhancement near mass threshold in $e^{+} e^{-} \rightarrow \Lambda \bar{\Lambda}$, Phys. Rev. D 97, 032013 (2018), doi:10.1103/PhysRevD.97.032013.

[28] G. Pakhlova et al., Observation of a near-threshold enhancement in the $e^{+} e^{-} \rightarrow \Lambda_{c}^{+} \bar{\Lambda}_{c}^{-}$ cross section using initial-state radiation, Phys. Rev. Lett. 101, 172001 (2008), doi:10.1103/PhysRevLett.101.172001.

[29] M. Ablikim et al., Precision measurement of the $e^{+} e^{-} \rightarrow \Lambda_{c}^{+} \bar{\Lambda}_{c}^{-}$cross section near threshold, Phys. Rev. Lett. 120, 132001 (2018), doi:10.1103/PhysRevLett.120.132001.

[30] F. A. Harris et al., BEPCII and BESIII, Int. J. Mod. Phys. A 24, 377 (2009), doi:10.1142/S0217751X09043705.

[31] M. Ablikim et al., Design and construction of the BESIII detector, Nucl. Instrum. Methods Phys. Res. A: Accel. Spectrom. Detect. Assoc. Equip. 614, 345 (2010), doi:10.1016/j.nima.2009.12.050.

[32] M. Ablikim et al., Complete measurement of the $\Lambda$ electromagnetic form factors, Phys. Rev. Lett. 123, 122003 (2019), doi:10.1103/PhysRevLett.123.122003.

[33] G. Fäldt, Polarization observables in the $e^{+} e^{-} \rightarrow \bar{\Lambda} \Lambda$ reaction, Eur. Phys. J. A 52, 141 (2016), doi:10.1140/epja/i2016-16141-y.

[34] G. Fäldt and A. Kupsc, Hadronic structure functions in the $e^{+} e^{-} \rightarrow \Lambda \bar{\Lambda}$ reaction, Phys. Lett. B 772, 16 (2017), doi:10.1016/j.physletb.2017.06.011.

[35] M. Ablikim et al., Study of the process $e^{+} e^{-} \rightarrow p \bar{p}$ via initial state radiation at BESIII, Phys. Rev. D 99, 092002 (2019), doi:10.1103/PhysRevD.99.092002.

[36] J. Haidenbauer and U.-G. Meißner, The electromagnetic form factors of the $\Lambda$ in the timelike region, Phys. Lett. B 761, 456 (2016), doi:10.1016/j.physletb.2016.08.067.

[37] J. Haidenbauer, T. Hippchen, K. Holinde, B. Holzenkamp, V. Mull and J. Speth, Reaction $p \bar{p} \rightarrow \Lambda \bar{\Lambda}$ in the meson-exchange picture, Phys. Rev. C 45, 931 (1992), doi:10.1103/PhysRevC.45.931.

[38] E. Klempt, F. Bradamante, A. Martin and J.-M. Richard, Antinucleon-nucleon interaction at low energy: scattering and protonium, Phys. Rep. 368, 119 (2002), doi:10.1016/S0370-1573(02)00144-8.

[39] J. Adamczewski-Musch et al., Proposal for experiments at SIS18 during FAIR Phase 0 (2017). 
[40] M. Kobayashi and T. Maskawa, CP-violation in the renormalizable theory of weak interaction, Prog. Theor. Phys. 49, 652 (1973), doi:10.1143/PTP.49.652.

[41] N. Cabibbo, Unitary symmetry and leptonic decays, Phys. Rev. Lett. 10, 531 (1963), doi:10.1103/PhysRevLett.10.531.

[42] The Particle Data Group, Chapter 13. CP violation in the quark sector, Phys. Rev. D 98, 030001 (2018), doi:10.1103/PhysRevD.98.030001 [Update 2019 http://pdg.lbl.gov/2019/reviews/rpp2018-rev-cp-violation.pdf].

[43] R. Aaij et al., Observation of CP violation in charm decays, Phys. Rev. Lett. 122, 211803 (2019), doi:10.1103/PhysRevLett.122.211803.

[44] R. Aaij et al., Measurement of matter-antimatter differences in beauty baryon decays, Nat. Phys. 13, 391 (2017), doi:10.1038/nphys4021.

[45] L. Canetti, M. Drewes and M. Shaposhnikov, Matter and antimatter in the universe, New J. Phys. 14, 095012 (2012), doi:10.1088/1367-2630/14/9/095012.

[46] X.-G. He, H. Murayama, S. Pakvasa and G. Valencia, CP violation in hyperon decays from supersymmetry, Phys. Rev. D 61, 071701 (2000), doi:10.1103/PhysRevD.61.071701.

[47] J. Tandean and G. Valencia, CP violation in hyperon nonleptonic decays within the standard model, Phys. Rev. D 67, 056001 (2003), doi:10.1103/PhysRevD.67.056001.

[48] J. F. Donoghue, X.-G. He and S. Pakvasa, Hyperon decays and CP nonconservation, Phys. Rev. D 34, 833 (1986), doi:10.1103/PhysRevD.34.833.

[49] T. Holmstrom et al., Search for $C P$ violation in charged $\Xi$ and $\Lambda$ hyperon decays, Phys. Rev. Lett. 93, 262001 (2004), doi:10.1103/PhysRevLett.93.262001.

[50] M. Ablikim et al., Polarization and entanglement in baryon-antibaryon pair production in electron-positron annihilation, Nat. Phys. 15, 631 (2019), doi:10.1038/s41567-0190494-8.

[51] J. W. Cronin and O. E. Overseth, Measurement of the decay parameters of the $\Lambda^{0}$ particle, Phys. Rev. 129, 1795 (1963), doi:10.1103/PhysRev.129.1795.

[52] O. E. Overseth and R. F. Roth, Time reversal Invariance in $\Lambda^{0}$ decay, Phys. Rev. Lett. 19, 391 (1967), doi:10.1103/PhysRevLett.19.391.

[53] P. M. Dauber, J. P. Berge, J. R. Hubbard, D. W. Merrill and R. A. Muller, Production and decay of cascade hyperons, Phys. Rev. 179, 1262 (1969), doi:10.1103/PhysRev.179.1262.

[54] W. E. Cleland, G. Conforto, G. H. Eaton, H. J. Gerber, M. Reinharz, A. Gautschi, E. Heer, C. Revillard and G. Von Dardel, A measurement of the $\beta$-parameter in the charged nonleptonic decay of the $\Lambda^{0}$ hyperon, Nucl. Phys. B 40, 221 (1972), doi:10.1016/05503213(72)90544-5.

[55] P. Astbury, J. Gallivan, J. Jafar, M. Letheren, V. Steiner, J. A. Wilson, W. Beusch, M. Borghini, D. Websdale, L. Fluri, K. Freudenreich F. X. Gentit, W. Wetzel, P. Le Du and O. Guisan, Measurement of the differential cross section and the spin-correlation parameters $P, A$ and $R$ in the backward peak of $\pi^{-} p \rightarrow K^{0} \Lambda$ at $5 \mathrm{GeV} / \mathrm{c}$, Nucl. Phys. B 99, 30 (1975), doi:10.1016/0550-3213(75)90054-1.

[56] P. Adlarson and A. Kupsc, CP symmetry tests in the cascade-anticascade decay of charmonium, Phys. Rev. D 100, 114005 (2019), doi:10.1103/PhysRevD.100.114005. 
[57] G. Fäldt and K. Schönning, Sequential hyperon decays in the reaction $e^{+} e^{-} \rightarrow \Sigma^{0} \bar{\Sigma}^{0}$ (2019), arXiv:1908.04157.

[58] S. S. Nair, E. Perotti and S. Leupold, Constraining $P$ and $C P$ violation in the main decay of the neutral Sigma hyperon, Phys. Lett. B 788, 535 (2019), doi:10.1016/j.physletb.2018.09.065.

[59] PANDA Collaboration, PANDA technical progress report, FAIR-ESAC (2005).

[60] T. Johansson, Antihyperon-hyperon production in antiproton-proton collisions, AIP Conf. Proc. 796, 95 (2005), doi:10.1063/1.2130143.

[61] W. Ikegami-Andersson, Future spin observables measurements with the PANDA detector at FAIR, Proceedings of the 6th International Workshop for Next Generation Scientists (FAIRNESS 2019), Arenzano, Italy, May 20-24 (2019).

[62] G. Perez Andrade, Production of the $\Sigma^{0}$ hyperons in the PANDA experiment at FAIR, Master thesis, Uppsala University (2019).

[63] S. Spataro et al., The PandaRoot framework for simulation, reconstruction and analysis, J. Phys.: Conf. Ser., 331, 032031 (2011), doi:10.1088/1742-6596/331/3/032031.

[64] H. Becker et al., Measurement of the reactions $\bar{p} p \rightarrow \bar{\Lambda} \Lambda, \bar{p} p \rightarrow \bar{\Lambda} \Sigma^{0}$ and $\bar{p} p \rightarrow \bar{\Lambda}$ (missing mass) at $6 \mathrm{GeV}$, Nucl. Phys. B 141, 48 (1978), doi:10.1016/0550-3213(78)90333-4.

[65] A. B. Kaidalov and P. E. Volkovitsky, Binary reactions in $\bar{p} p$ collisions at intermediate energies, Z. Phys. C 63, 517 (1994), doi:10.1007/BF01580332. 